\title{
Abordagem restauradora estética e conservadora para o fechamento de diastemas múltiplos após tratamento ortodôntico: relato de caso
}

\begin{abstract}
- Cassiana Koch Scott Departamento de Dentística, Endodontia e Materiais Dentários, Faculdade de Odontologia de Bauru, Universidade de São Paulo, Bauru, SP, Brasil - Marilia Mattar de Amoêdo Campos Velo Departamento de Dentística, Endodontia e Materiais Dentários, Faculdade de Odontologia de Bauru, Universidade de São Paulo, Bauru, SP, Brasil • Martha Beteguelli Michellin Departamento de Dentística, Endodontia e Materiais Dentários, Faculdade de Odontologia de Bauru, Universidade de São Paulo, Bauru, SP, Brasil • Giovanna Speranza Zabeu Departamento de Dentística, Endodontia e Materiais Dentários, Faculdade de Odontologia de Bauru, Universidade de São Paulo, Bauru, SP, Brasil • Ernesto Benalcázar Jalkh Departamento de Reabilitação Oral, Faculdade de Odontologia de Bauru, Universidade de São Paulo, Bauru, SP, Brasil - Juliana Fraga Soares Bombonatti Departamento de Dentística, Endodontia e Materiais Dentários, Faculdade de Odontologia de Bauru, Universidade de São Paulo, Bauru, SP, Brasil • Rafael Francisco Lia Mondelli Departamento de Dentística, Endodontia e Materiais Dentários, Faculdade de Odontologia de Bauru, Universidade de São Paulo, Bauru, SP, Brasil
\end{abstract}

RESUMO | Objetivos: A presença de diastemas múltiplos na região anterior compromete a estética do sorriso e a harmonia da face, podendo interferir severamente na qualidade de vida do paciente. Este relato clínico tem como objetivo abordar uma técnica restauradora direta e conservadora para a resolução estética de diastemas anteriores múltiplos, empregando resina composta e abordando os princípios restauradores para maior previsibilidade de resultado. Materiais e métodos: O tratamento consistiu no clareamento caseiro com gel à base de peróxido de carbamida 16\% durante 14 dias por três horas diárias. Após moldagem e obtenção dos modelos de gesso, o enceramento de diagnóstico foi realizado e a guia palatina em silicone de condensação, obtida. As restaurações diretas em resina composta para o fechamento dos diastemas foram realizadas na cor E-bleaching L, D-bleaching e T-neutral (Opalis, FGM, Joinville, SC, Brasil), proporcionando o reposicionamento da linha média e conferindo adequada inclinação e alinhamento aos dentes anteriores. Após sete dias foi realizado o acabamento, RX final, remoção dos excessos e polimento das restaurações. Resultados: o controle clínico foi realizado periodicamente, mostrando resultado satisfatório após um ano e oito meses de acompanhamento. Conclusões: o conhecimento em relação aos materiais restauradores diretos e o domínio da técnica operatória promovem maior previsibilidade e melhor desempenho clínico das restaurações estéticas em resinas compostas.

DESCRITORES | Diastema; Resina Composta; Estética Dentária.

ABSTRACT | Aesthetic and conservative restorative approach for multiple diastema closure after orthodontic treatment: a case report • Objectives: The presence of multiple diastema in anterior teeth compromises the smile esthetics and the harmony of the face, often interfering in the quality of life of the patient. This case report aims to address a direct and conservative restorative technique with composite resin for the esthetic resolution of multiple anterior diastema, reporting the restorative principles and indications for greater outcome predictability. Materials and methods: The treatment consisted of at-home whitening with $16 \%$ carbamide peroxide gel in a custom-fitted tray for 14 days. After obtaining the impressions, the gypsum models were obtained, and the wax-up and palatal silicone guide were performed. The direct composite resin restorations for diastema closure were performed utilizing the shades E-bleaching $\mathrm{L}$, D-bleaching and T-neutral (Opalis, FGM, Joinville, Brasil), providing repositioning of the midline, adequate inclination and alignment of the anterior teeth. Results: After 7 days the restorations were finished and polished and final X Ray was obtained. Conclusion: Based on the results after 1 year and 8 months, it is important to know the relationship between direct restorative materials and the domain of operative technique in order to provide greater predictability and satisfactory aesthetic results.

DESCRIPTORS | Diastema; Composite Resin; Dental Esthetics.

AUTOR CORRESPONDENTE | - Cassiana Koch Scotti Departamento de Dentística, Endodontia e Materiais Dentários, Faculdade de Odontologia de Bauru, Universidade de São Paulo, Bauru, SP, Brasil • Al. Octávio Pinheiro Brisolla, 9-7 Bauru, SP, Brasil • 517012-901 E-mail: cassikoch@hotmail.com

- Received Aug. 28, 2018 • Accepted Jan. 15, 2019

- Dol http://dx.doi.org/10.11606/issn.2357-8041.clrd.2019.149527 


\section{INTRODUÇÃO}

Prover tratamentos odontológicos que estejam atrelados à saúde e à função e que estejam alinhados a parâmetros estéticos de alto nível representa, atualmente, o ponto-chave para exercer uma odontologia de excelência. Entretanto, é imperativo que o profissional adote um planejamento multidisciplinar adequado, permitindo assim resultados reabilitadores e estéticos de alta qualidade, mais conservadores e previsíveis. ${ }^{1,2}$ Neste contexto, é frequente nos consultórios odontológicos a busca por tratamento visando à resolução de diastemas nos dentes anteriores superiores, os quais na maioria das vezes requerem abordagens que englobam diferentes áreas da Odontologia, destacando-se a Ortodontia, a Dentística restauradora e, por vezes, a Periodontia. ${ }^{1,3,4}$

Os diastemas dentários ocorrem frequentemente na população adulta, afetando em grande proporção o gênero feminino e pacientes asiáticos. Geralmente sua etiologia é de caráter multifatorial, podendo estar relacionada às anomalias dentárias de forma $\mathrm{e}$ tamanho, bem como pela discrepância dentoalveolar de Bolton. ${ }^{5}$ No entanto, outros fatores também podem estar envolvidos no desenvolvimento dos diastemas, como as características hereditárias, hábitos deletérios, desequilíbrio muscular, migração dentária fisiológica ou patológica, agenesia dentária, odontomas, cistos, fissuras palatinas, trespasse horizontal acentuado, defeitos na sutura intermaxilar e inserção do freio labial próximo a margem genvigal. ${ }^{6}$

O tratamento dos diastemas dentários baseiase em um planejamento minucioso e individual de cada caso e, em algumas situações clínicas, abordagens exclusivamente ortodônticas podem ser realizadas. Quando o tamanho e volume dos dentes são reduzidos e o tratamento é realizado apenas às custas de movimentação ortodôntica ocorre perda de volume na face vestibular, hipotonicidade muscular do lábio superior e acentuação do sulco nasogeniano. Consequentemente, a estética facial pode ser severamente prejudicada., ${ }^{7}$

Dessa forma, as restaurações diretas em resina composta representam uma ferramenta biomimética e conservadora para o tratamento dos diastemas dentários. Frequentemente, essas restaurações não necessitam de preparo e desgaste prévio da estrutura dentária, pois apresentam comportamento promissor da interface adesiva, estética altamente satisfatória e longevidade clínica, além de possuírem um custo acessível ao paciente. ${ }^{9}$ Por outro lado, os laminados cerâmicos também são considerados uma alternativa de tratamento para o fechamento dos diastemas dentários. Entretanto, trata-se de uma opção que muitas vezes exige desgaste dos dentes para promover espaço e eixo de inserção, maior número de sessões clínicas e maior custo. Além disso, depende da habilidade e comunicação com o técnico de laboratório e é de difícil reparo.9,10

Diante disso, a abordagem restauradora em resina composta é considerada uma boa opção principalmente para pacientes jovens em virtude do maior conservadorismo. Um diagnóstico preciso associado à um adequado plano de tratamento, considerando uma análise detalhada da oclusão, do sorriso, incluindo fotos faciais e intraorais, planejamento digital e enceramento diagnóstico são indispensáveis para uma correta abordagem restauradora. ${ }^{11}$

Assim, o presente relato clínico visa discutir os princípios estéticos para um plano de tratamento multidisciplinar. O tratamento incluiu o clareamento de moldeira sob supervisão seguido da confecção de restaurações diretas em resina composta nos elementos 11, 12, 13, 21, 22 e 23, após tratamento ortodôntico realizado durante três anos.

\section{RELATO DO CASO}

Paciente jovem do gênero masculino, 18 anos de idade, procurou atendimento odontológico no Departamento de Dentística, Endodontia 
e Materiais Odontológicos, na Faculdade de Odontologia de Bauru, queixando-se de insatisfação estética e acentuado comprometimento da qualidade de vida e relacionamento interpessoal, em virtude da presença de diastemas nos dentes anteriores superiores (Figuras 1 a 3). Durante a anamnese, o paciente relatou recente finalização do tratamento ortodôntico, que teve duração de três anos e dois meses e nenhuma contraindicação para a realização do clareamento de moldeira sob supervisão. Na primeira sessão, foi conduzido um exame clínico detalhado e checagem da oclusão, verificando-se as guias de desoclusão e o correto posicionamento dos caninos e primeiros molares em classe I de Angle, ausência de contatos nos dentes posteriores nos movimentos de lateralidade, guia anterior no movimento de protrusão, ausência de interferências oclusais, posicionamento da linha média dentária em relação a linha média facial, como também verificou-se a remoção de todos os excessos de resina utilizada na colagem dos bráquetes ortodônticos.
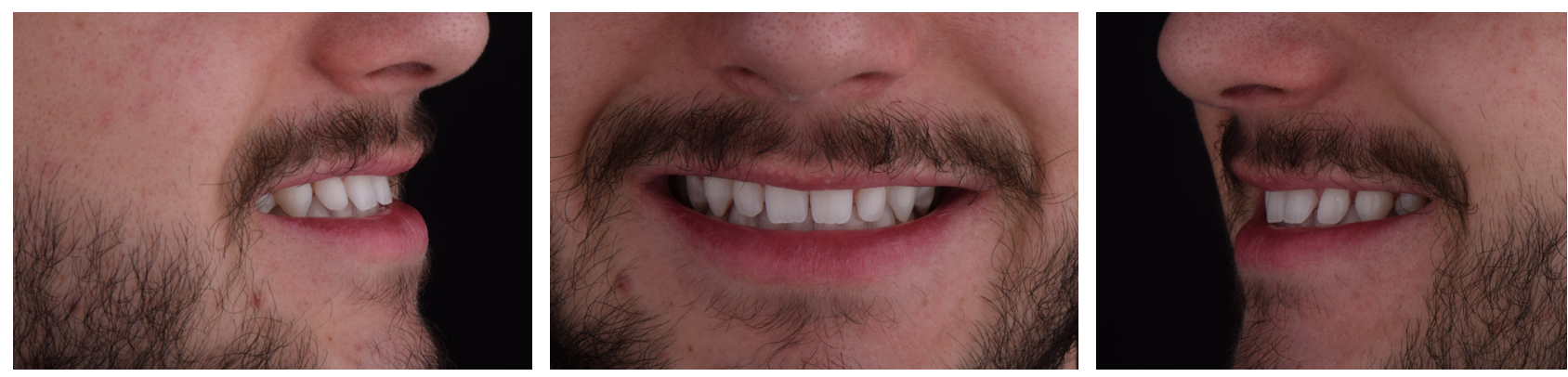

Figuras 1-3 | Diastemas nos dentes anteriores superiores.

Foram realizadas as fotos iniciais intra e extraorais padronizadas para a realização do planejamento digital do sorriso orientado pela grade de proporção áurea digital (Figura 4). Na sequência, foram realizadas duas moldagens dos arcos dentários superior e inferior com alginato (Cavex Cream Alginate, Cavex Holland BV, Haarlem, Holanda) cujos modelos foram obtidos em gesso pedra especial tipo

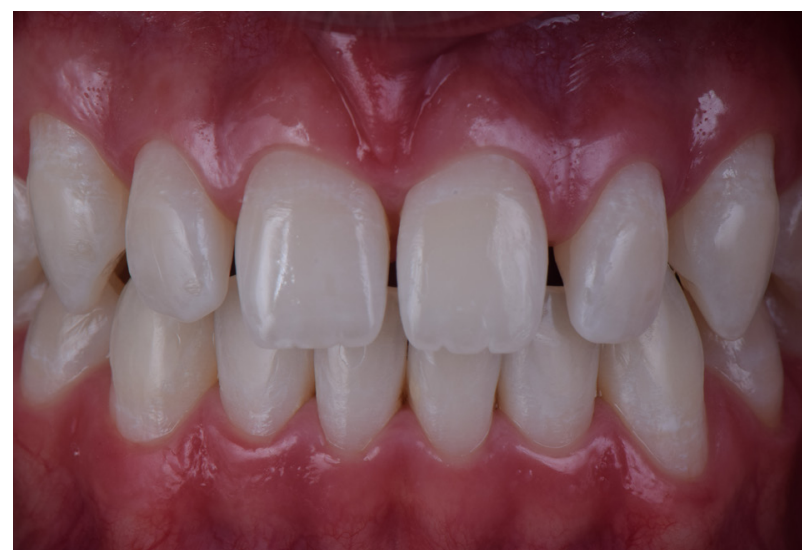

Figura 4 | Imagem para a realização do planejamento digital.
IV (Gesso tipo IV, Durone, Dentsply, Rio de Janeiro, RJ, Brasil). Dois modelos (inferior e superior) foram destinados à confecção das moldeiras individuais para o clareamento pela técnica caseira e outros dois modelos foram utilizados para a confecção do enceramento diagnóstico dos dentes 11, 12, 13, 21, 22 e 23, considerando a proporção regressiva de aparecimento determinada no planejamento digital (Figura 5a e 5b).

Em uma segunda sessão, foi realizado o registro de cor dos dentes do paciente (cor A2), e as orientações ao paciente para o início do clareamento caseiro prévio ao tratamento restaurador foram realizadas, com gel a base de peróxido de carbamida a 16\% Whiteness Perfect (FGM, Joinville, SC, Brasil), com protocolo de três horas por dia durante 14 dias. Após um período de sete dias do término do clareamento, a cor estabilizou em BL 2 na escala de cor A-D Shade Guide Bleach (Ivoclar Vivadent, Schaan, Liechtenstein). 


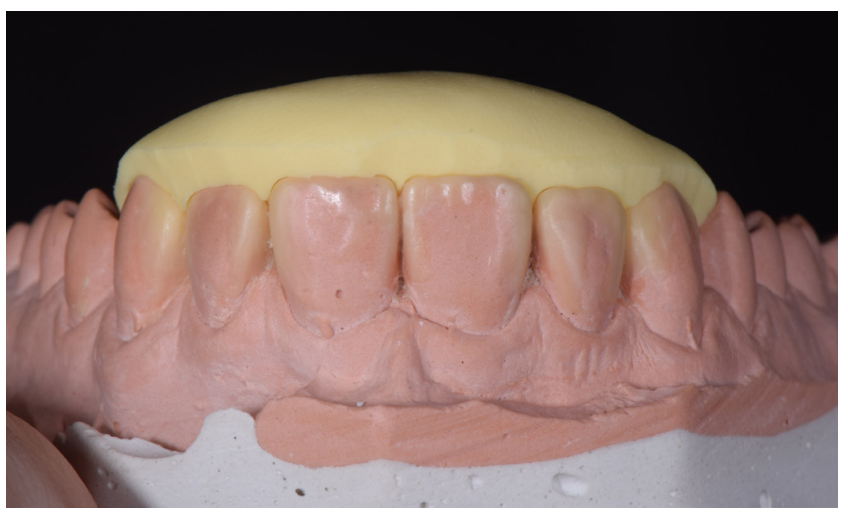

Figura 5 | Modelo utilizado para a confecção do enceramento.

$\mathrm{Na}$ fase restauradora, inicialmente uma matriz de silicone de condensação (Optosil Confort Putty Kit, Zulzer, São Paulo, SP, Brasil) foi confeccionada a partir do enceramento diagnóstico. Para checagem da adaptação, a guia de silicone foi levada em posição na cavidade bucal. Sequencialmente, foi realizada a seleção de cor, cuja croma foi determinada pela inserção de pequenos incrementos de resina de características menos translúcidas (resinas para dentina) e polimerizadas sobre a superfície vestibular no terço cervical de cada dente. A seleção de luminosidade e cor da resina de característica mais translúcida (para esmalte) foi realizada através da inserção de pequenos incrementos polimerizados no terço incisal, os quais foram comparados em foto preto e branco para verificação do valor do dente e da resina. As resinas compostas selecionadas foram as de cor E-bleaching L, D-bleaching e T-neutral (Opalis, FGM, Joinville, SC, Brasil).

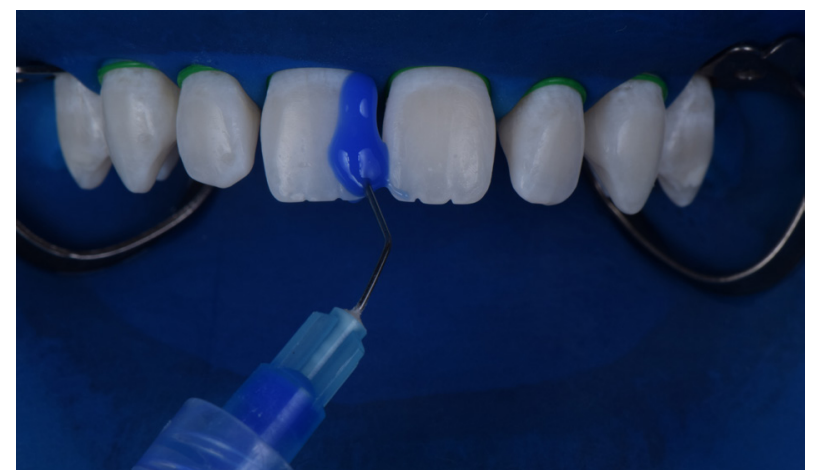

Figura 6 | Uso de borrachas ortodônticas individualizadas para cada dente.

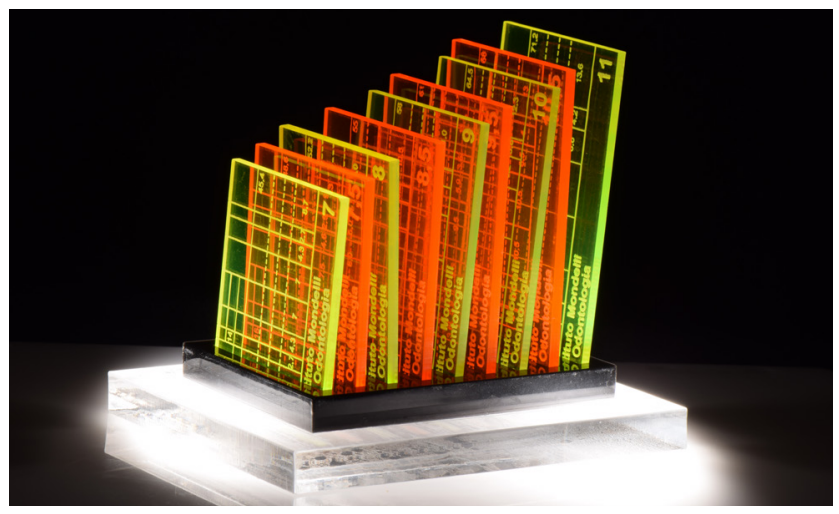

Após a seleção de cor, foi realizado o isolamento absoluto do campo operatório com dique de borracha (Madeitex, São José dos Campos, SP, Brasil) estabilizado por grampos n 29 nos primeiros prémolares superiores, cujo afastamento gengival foi otimizado pelo uso de borrachas ortodônticas individualizadas para cada dente (Figura 6). Com o campo operatório devidamente limpo e seco, realizou-se a asperização da superfície do esmalte vestibular com ponta diamantada $n^{\circ}$ 2135 (Kg Sorense, Cotia, SP, Brasil) e iniciou-se o procedimento adesivo, através do condicionamento da superfície vestibular, proximal e lingual dos dentes concomitantemente com ácido fosfórico a $37 \%$ durante 30 segundos, considerando que o procedimento adesivo se restringia apenas ao esmalte dentário. Para garantir condicionamento total da superfície, o ácido fosfórico foi levado ao interior do sulco gengival através do uso de fio dental (Figura 7).

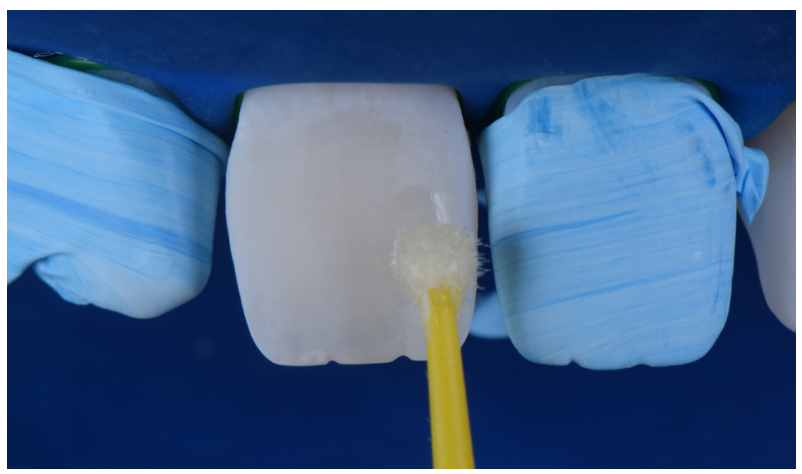

Figura 7 | Asperização da superfície do esmalte vestibular com ponta diamantada $n^{\circ} 2135$ para iniciar o procedimento adesivo. 
Posteriormente, os dentes adjacentes foram protegidos com fita para isolamento IsoTape (TDV, Pomerode, SC, Brasil) e o componente hidrofóbico (Scotchbond Multipurpose, 3M ESPE, São Paulo, SP, Brasil) do adesivo foi aplicado com microaplicador KG Brush (KG Sorensen, Cotia, SP, Brasil) e levado ao nível cervical com fio dental para garantir que toda área de esmalte condicionada fosse coberta pelo adesivo (Figura 7). Após a remoção dos excessos com leve jato de ar, o adesivo foi polimerizado por 20 segundos, utilizando uma unidade fotoativadora de luz LED (>100omW/cm² D700; Dabi Atlante, São Paulo, SP, Brasil). Esse procedimento foi repetido em cada dente a ser restaurado, antes da inserção da resina composta.

Sequencialmente, uma fina camada de aproximadamente 0,3 milímetros de resina para esmalte foi inserida sobre a face palatina impressa na guia de silicone palatina (Figuras 8 e 9). Neste momento, é importante garantir a adaptação do material restaurador no nível cervical, bem como a separação dos dentes adjacentes que podem aderir por extravasamento de adesivo ou material restaurador na face proximal (Figura 10).

Para mimetizar os mamelos e conferir o croma incisal, foram inseridos pequenos incrementos de resina composta de dentina, acomodados com sonda exploradora para reprodução da caracterização intrínseca da dentina que contribui na reflexão da luz quando incidida sobre o material (Figura 11). É importante garantir nesta etapa a sobreposição completa do terço incisal do dente pelo incremento de resina para dentina, de modo a mascarar a borda incisal do dente pelo uso do material restaurador. Foi inserida resina de efeito (T-Neutral) entre os mamelos para evidenciá-los (Figuras 12 e 13). Ao longo de toda a técnica incremental, a fotoativação foi realizada em incrementos de no máximo 2,0 milímetros para promover adequado grau de conversão dos monômeros, bem como para permitir as seguintes etapas da estratificação.
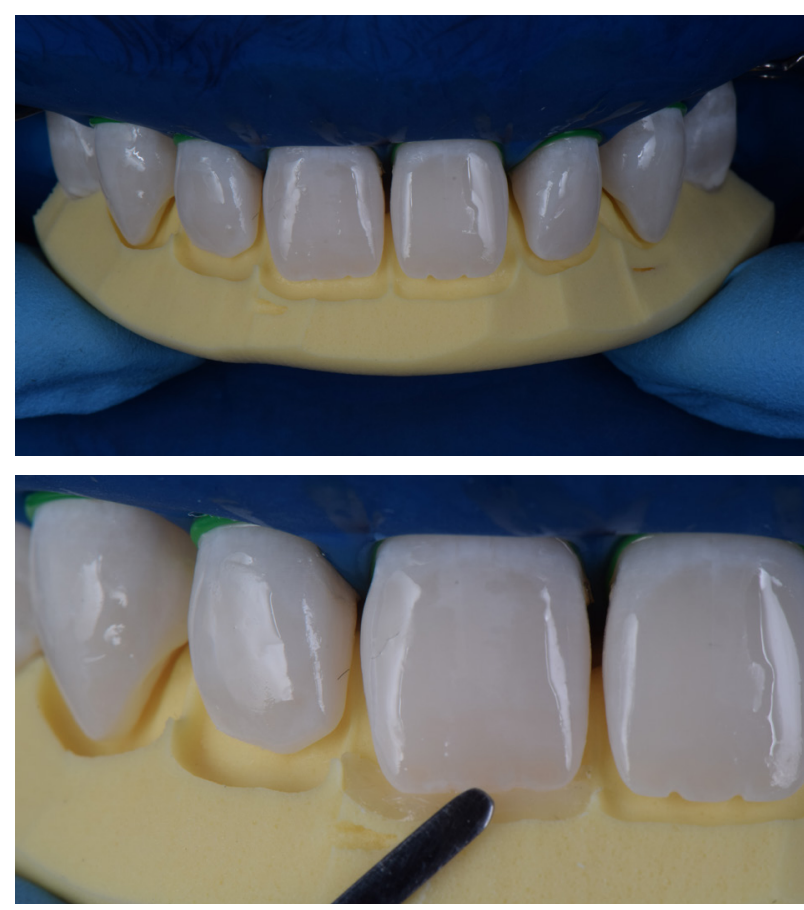

Figuras 8-9 | Camada de resina para esmalte foi inserida sobre a face palatina impressa na guia de silicone palatina.

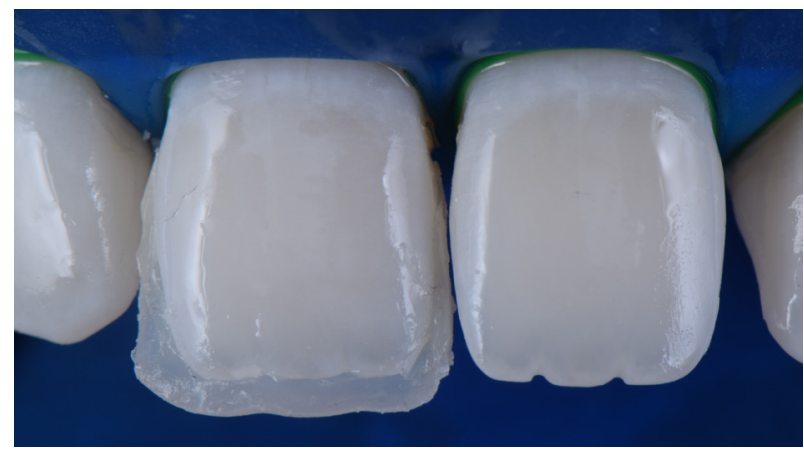

Figura 10 || Importante garantir a adaptação do material restaurador no nível cervical e a separação dos dentes adjacentes que podem aderir por extravasamento de adesivo ou material restaurador na face proximal.

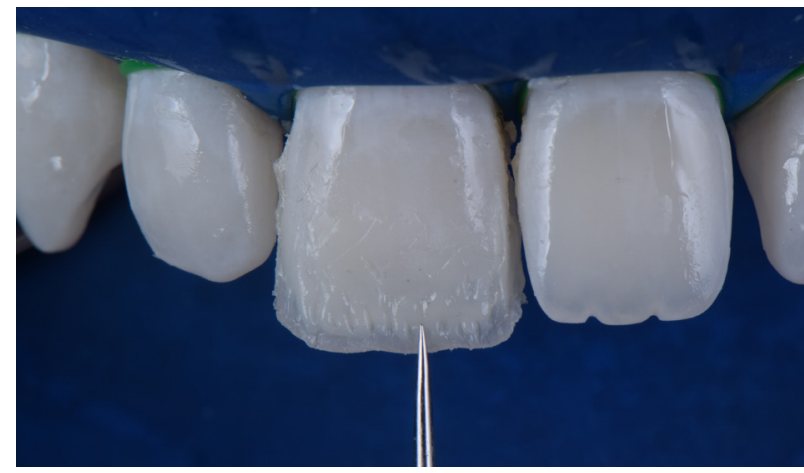

Figura 11 || Para mimetizar os mamelos e conferir o croma incisal, insere-se pequenos incrementos de resina composta de dentina. 

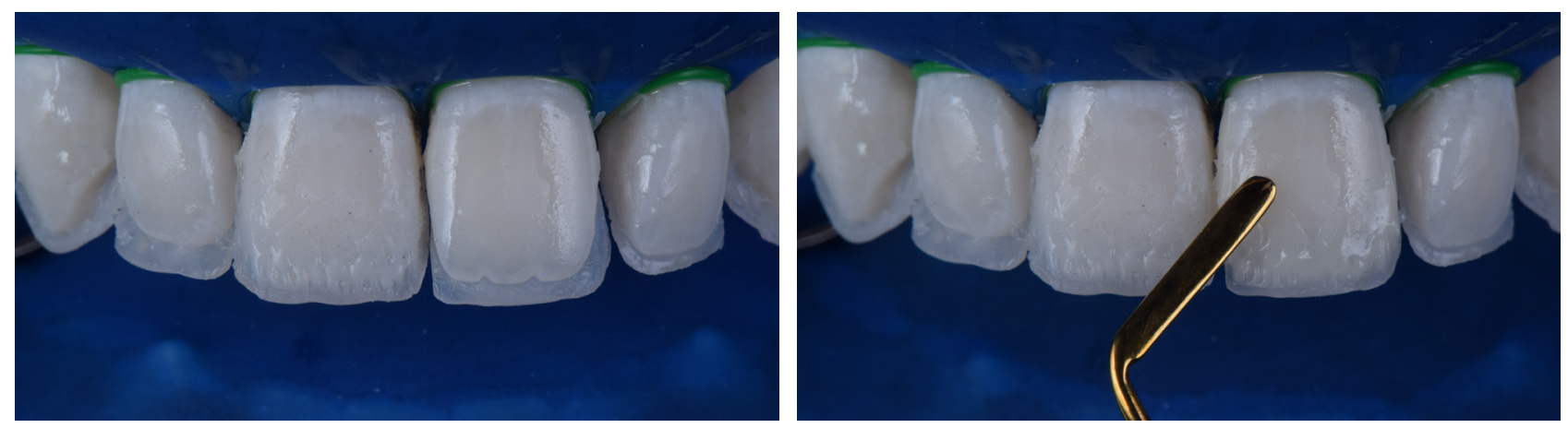

Figuras 12-13 || Inserção de resina de efeito (T-Neutral) entre os mamelos.

As faces proximais foram desenvolvidas com resina para esmalte, garantindo adequado posicionamento do ponto de contato e, sequencialmente, foi inserida uma única camada fina de resina para esmalte, acomodada com pincel sobre toda a superfície vestibular (Figuras 14 e 15). Ao finalizar a restauração, foi utilizada uma cerra metálica fina entre as faces proximais (Figura 16). O acabamento imediato foi realizado com lâmina cirúrgica \#12 D (Lamedid, Barueri, SP, Brasil), brocas multilaminadas
(KG Sorense, São Paulo, SP, Brasil) e discos abrasivos (Sof-lex, 3M ESPE, São Paulo, SP, Brasil) sob isolamento absoluto (Figura 17). O perfil das restaurações foi verificado por vista incisal, proximal e na posição de 12 horas, avaliando a inclinação vestibular dos dentes restaurados, volume vestibular, adaptação da face palatina e reprodução dos acidentes anatômicos. Os contatos oclusais foram checados em Máxima Intercuspidação Habital (MIH) e nos movimentos excêntricos de lateralidade e protrusão.
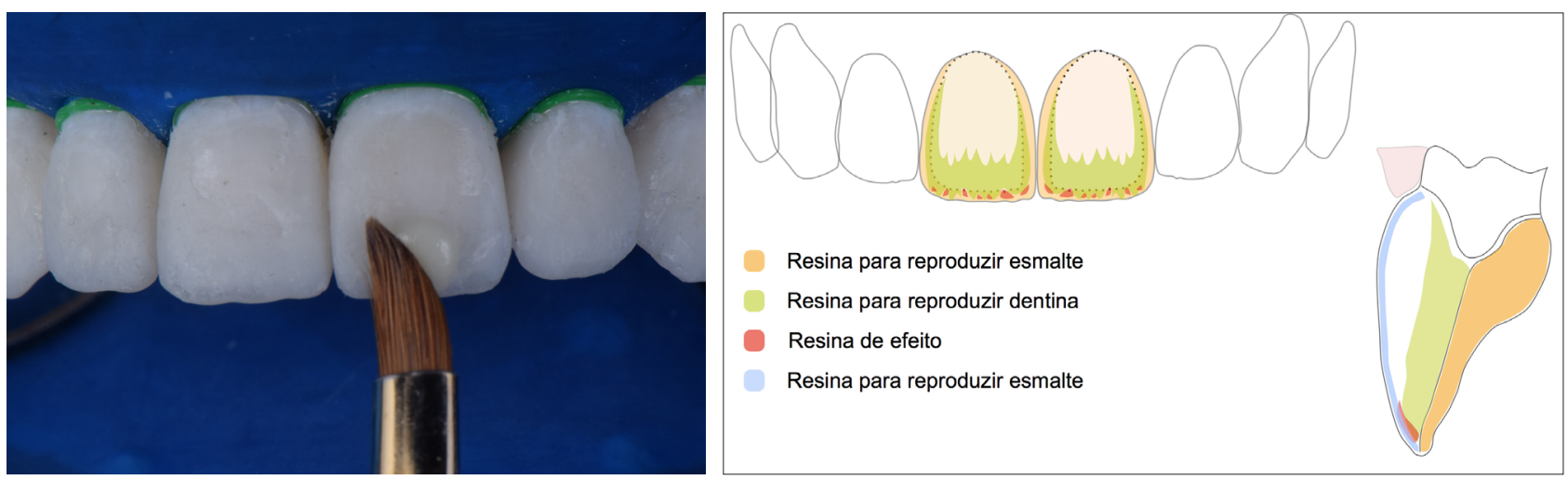

Figuras 14-15 | Fina de resina para esmalte, acomodada com pincel sobre toda a superfície vestibular.

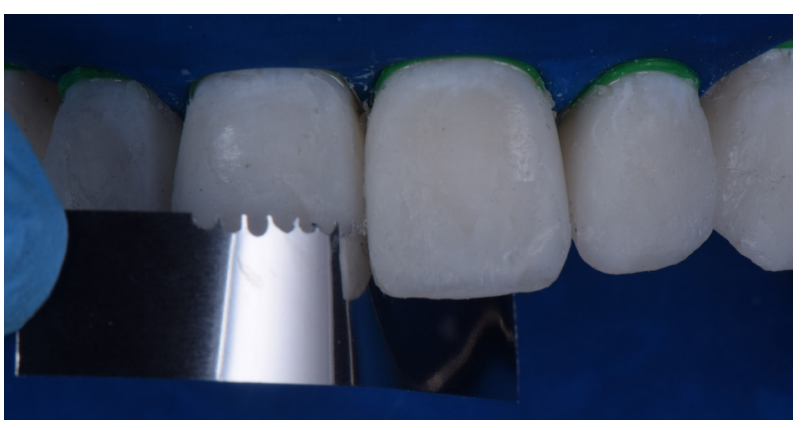

Figura 16 | Utilização de cerra metálica fina entre as faces proximais.

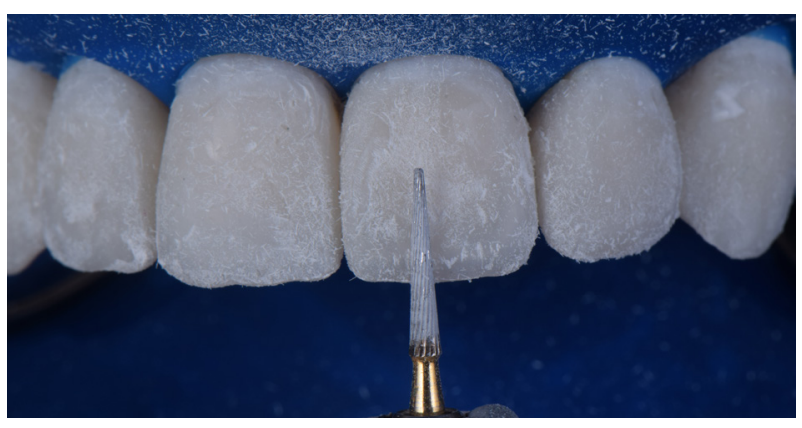

Figura 17 | O acabamento realizado com lâmina cirúrgica \#12 D, brocas multilaminadas e discos abrasivos. 
Após 48 horas, o acabamento refinado e o polimento das restaurações foram realizados utilizando sequência de disco de lixa abrasivos para polimento (Sof-lex, 3M ESPE, São Paulo, SP, Brasil) (Figuras 18 e 19), borrachas abrasivas siliconadas na sequência de 3 granulações (Jiffy, Ultradent, Indaiatuba, SP, Brasil) (Figura 20) e escova de carbeto de silício (Astrobrush CA - Ivoclar Vivadent, Schaan, Liechtenstein) em baixa rotação (Figura 21).

O resultado final é observado na figura 22, constatando a integração dos procedimentos estéticos com as expectativas do paciente, estando em harmonia com a face e alinhado com os parâmetros de manutenção da saúde e função.
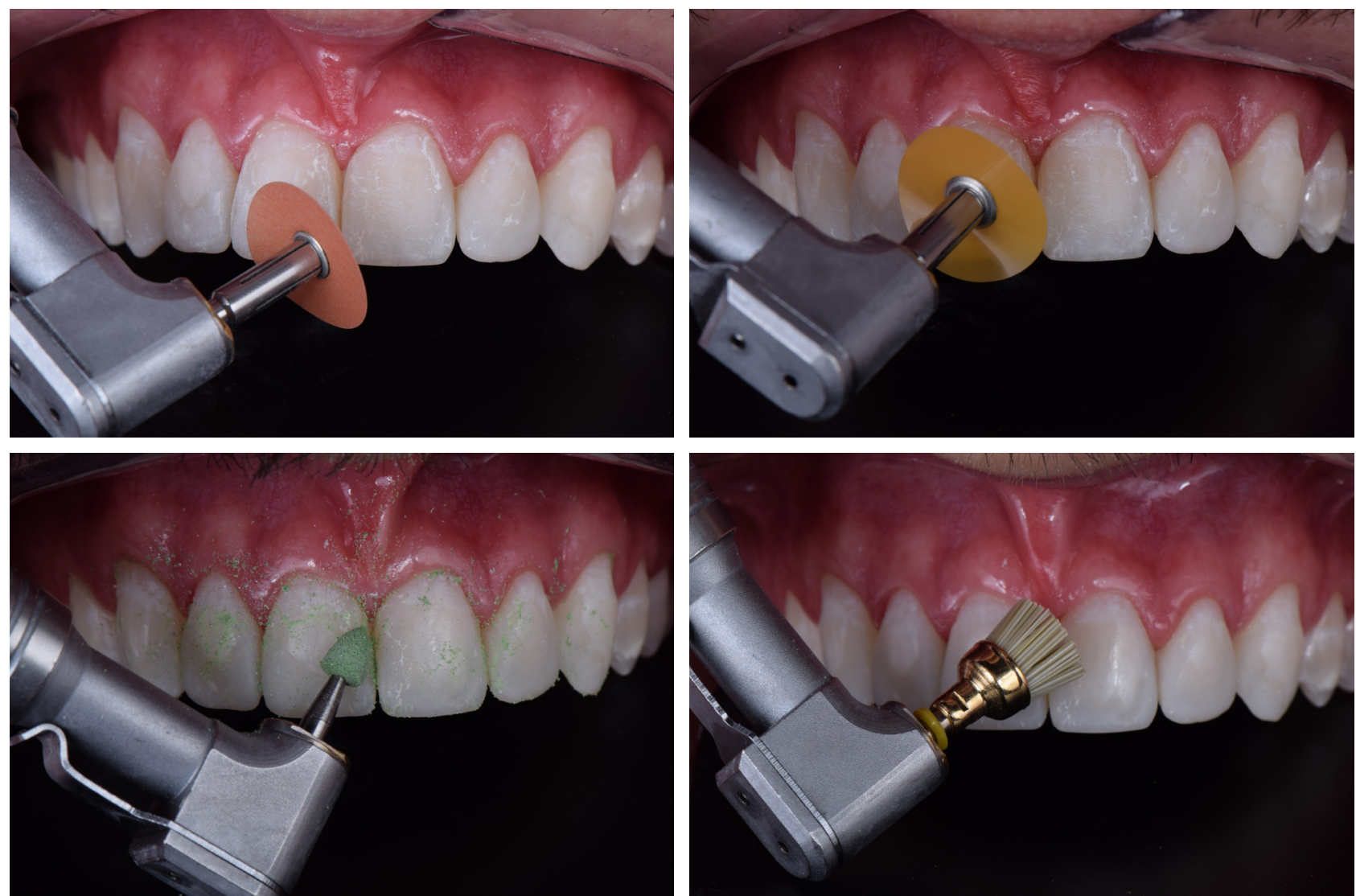

Figuras 18-21 | Após 48 horas, acabamento utilizando sequência de disco de lixa abrasivos para polimento, borrachas abrasivas siliconadas na sequência de 3 granulações e escova de carbeto de silício em baixa rotação.

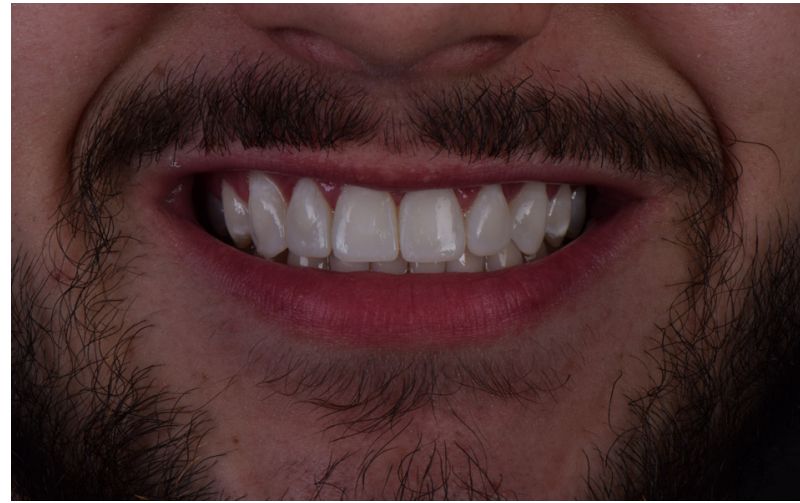

Figura 22 | Resultado.

\section{DISCUSSÃO}

Diante da dificuldade em planejar a distribuição e fechamento do diastema em dentes anteriores, sua resolução exclusiva através do tratamento ortodôntico é um grande desafio ao cirurgiãodentista e requer conhecimento dos parâmetros e princípios estéticos da face. Em muitas situações clínicas, em virtude do tamanho reduzido e do volume dos dentes anteriores do paciente, a redução dos espaços sem acréscimo de material restaurador 
invariavelmente traz resultados desarmônicos e pouco estéticos. ${ }^{4,5,10}$

O presente caso clínico mostrou a presença de diastema nos dentes anteriores decorrente de discrepâncias dentoalveolares. Geralmente nestes casos, o tratamento de eleição recai sobre restaurações diretas ou indiretas. Entretanto, em muitas situações clínicas, como no presente relato, o tratamento multidisciplinar se faz necessário, integrando o tratamento ortodôntico para restabelecer a proporção largura-altura, seguido por tratamentos estéticos (clareamento e restaurações). ${ }^{12}$

Para que um resultado final satisfatório fosse atingido, considerando a inclinação vestibular acentuada dos dentes do paciente após o tratamento ortodôntico, o enceramento de diagnóstico foi utilizado, uma vez que esta ferramenta auxilia o estabelecimento de padrões de referência de volume na face vestibular de material a ser acrescentado para que as características antiestéticas sejam corrigidas. $^{13}$

Objetivando a confecção de um enceramento diagnóstico individualizado, que englobe todos os aspectos faciais do sorriso do paciente, o planejamento digital, através de fotos faciais e intraorais, tem sido considerado um elementochave para aperfeiçoar o diagnóstico, para dar previsibilidade ao tratamento e para contribuir com o sucesso do procedimento restaurador. Com a aplicação do planejamento digital durante o enceramento, é possível avaliar fora da cavidade bucal do paciente alguns fatores como: tamanho, formato, proporção de altura e largura para cada dente e a proporção regressiva de aparecimento, a qual é transferida para a boca através de guias. Esta manobra operatória possibilita priorizar a atenção do cirurgião-dentista durante o procedimento restaurador para fatores decisivos como a seleção de cor, procedimentos adesivos, adaptação cervical, perfil de emergência, polimerização e manutenção de campo operatório limpo e seco. ${ }^{10-13}$

Em associação com estes avanços tecnológicos, o desenvolvimento de materiais restauradores e adesivos mais aprimorados também tem revolucionado a odontologia restauradora, possibilitando que estes sejam aderidos à superfície dentária sem qualquer preparo ou desgaste, tornando os procedimentos de mínima intervenção e altamente satisfatórios do ponto de vista estético. No entanto, é extremamente necessário que haja o domínio da técnica restauradora pelo cirurgião-dentista ${ }^{14-16}$, uma vez que os procedimentos adesivos são altamente sensíveis à técnica empregada e estão diretamente relacionados ao sucesso clínico das restaurações. Dessa forma, todo o processo adesivo deve ser conduzido de acordo com as instruções do fabricante, sem negligenciar nenhuma etapa clínica, além do conhecimento sobre a composição e microestrutura do substrato dentário no qual será realizado. ${ }^{17}$

Para a seleção do material compósito a ser utilizado, o material de escolha foi uma resina nanohíbrida, uma vez que os compósitos nanohíbridos e nanoparticulados apresentam propriedades mecânicas satisfatórias e por vezes bastantes semelhantes à estrutura dentária, como resiliência, padrão de desgaste, manutenção da lisura e resistência a fratura. Além disso, a contração de polimerização pode ser modulada através das técnicas de fotoativação e técnica incremental, de modo a favorecer o grau de conversão, o excelente polimento e a estabilidade de cor, com boas propriedades ópticas no que se refere aos fenômenos que mimetizam o comportamento da estrutura dentária como fluorescência, translucidez, opalescência, alta gama de cromas, diferentes saturações e variações de valor. ${ }^{17-20}$

A técnica incremental empregada na confecção das restaurações com resina composta permite 
a estratificação das cores, bem como reduz a contração de polimerização, possibilita maior grau de conversão e compensa parcialmente o estresse nas paredes cavitárias, permitindo maior controle na inserção dos incrementos e refinamento da escultura. Nesse sentido, é importante que o cirurgião-dentista se preocupe com a anatomia final e com a cor da restauração durante a inserção dos incrementos de resina composta à medida que a restauração é desenvolvida até a remoção dos excessos e finalização da restauração para proporcionar adequado perfil de emergência da restauração e compatibilidade com a saúde periodontal. Ao final, deve-se evitar desgaste excessivo de material após a colocação do último incremento de resina para esmalte pois implica em remoção completa ou de boa parte da resina mais translúcida, expondo uma região mais opaca que configura artificialidade à restauração. ${ }^{21-24}$

Até o presente momento, a literatura tem indicado alta longevidade clínica para restaurações em resina composta em dentes anteriores, com taxa anual de falha de $0 \%$ a $4,1 \%$. Diante do caso exposto, fica evidente que problemas estéticos como diastemas múltiplos são corrigidos com excelência por restaurações diretas em resina composta provendo também um efetivo desempenho clínico ao longo do tempo. ${ }^{9,25}$

\section{CONCLUSÃO}

O presente relato de caso clínico mostrou a importância de um tratamento multidisciplinar com a integração de Ortodontia e Dentística para fechamento de diastema em caso de discrepâncias dentoalveolares. A escolha de procedimento restaurador direto com resina composta vai ao encontro de um tratamento de mínima intervenção. Além disso, baseado em evidências, o uso de nanocompósitos mostrou-se uma alternativa segura, com resultados altamente satisfatórios. O adequado planejamento proporciona melhores e mais previsíveis resultados.

\section{REFERÊNCIAS}

1. Saha MK, Khatri M, Saha SG, Dubey S, Saxena D, Vijaywargiya $\mathrm{N}$, et al. Perception of acceptable range of smiles by specialists, general dentists and lay persons and Evaluation of different aesthetic paradigms. J Clin Diagn Res. 2017 Feb;11(2):25-8. doi: 10.7860/JCDR/2017/23359.9274.

2. Orr C. 'State of the art' in aesthetic dentistry. Br Dent J. 2016 Oct;221(8):435. doi: 10.1038/sj.bdj.2016.748.

3. Ishikiriama SK, Maenosono RM, Ishikiriama BLC, Mondelli RFL, Wang L. Tratamento restaurador integrado. Full Dentistry in Science. 2012;(3):208-15.

4. Pinto RC, Chambrone L, Colombini BL, Ishikiriama SK, Britto IM, Romito GA. Minimally invasive esthetic therapy: a case report describing the advantages of a multidisciplinary approach. Quintessence Int. 2013 May;44(5):385-91. doi: 10.3290/j.qi.a29147.

5. Bolton WA. Disharmony in tooth size and its relation to the analysis and treatment of malocclusion. Angle. 1958 July;28(3):113-30.

6. Huang WJ, Creath CJ. The midline diastema: a review of its etiology and treatment. Pediatr Dent. 1995;17(3):171-9.

7. Willhite C. Diastema closure with freehand composite: controlling emergence contour. Quintessence Int. 2005 Feb:36(2):138-40.

8. Furuse AY, Baratto SS, Spina DR, Correr GM, da Cunha LF, Gonzaga CC. Planning extensive esthetic restorations for anterior teeth: use of waxed-up study casts and composite resin mock-ups. Gen Dent. 2016 Jan-Feb;64(1):6-9.

9. Laske M, Opdam NJ, Bronkhorst EM, Braspenning JC, Huysmans MC. Longevity of direct restorations in Dutch dental practive: descriptive study out of a practice based research network. J Dent. 2016;46:12-7. doi: 10.1016/j. jdent.2016.01.002.

10. Coachman C, Paravina RD. Digitally enhanced esthetic dentistry - From treatment planning to quality control. J Esthet Rest Dent. 2016 Mar; 28 Suppl 1:S3-4. doi: 10.1111/jerd.12205.

11. Abduo J. Geometrical effects of conventional and digital prosthodontic planning wax-ups on lateral oclusal contact number, contact area, and steepness. J Oral Sci. 2017;59(3):431-8. doi: 10.2334/josnusd.16-0610.

12. Kravitz ND. Smile analysis and design in the digital era. J Clin Orthod. 2017 Sep;51(9):602-5.

13. Coachman C, Calamita MA, Sesma N. Dynamic documentation of smile and the $2 \mathrm{D} / 3 \mathrm{D}$ digital smile design process. Int 
- Abordagem restauradora estética e conservadora para o fechamento de diastemas múltiplos após tratamento ortodôntico:

relato de caso

J Periodontics Restorative Dent. 2017 Mar-Apr;37(2):183-93. doi: 10.11607/prd.2911.

14. Pashley DH, Tay FR, Breschi L, Tjäderhane L, Carvalho RM, Carrilho M, Tezvergil-Mutluay A. State of the art etch-and-rinse adhesives. Dent Mater. 2011 Jan;27(1). doi: 10.1016/j. dental.2010.10.016.

15. Ahmed KE, Murbay S. Survival rates of anterior composites in managing tooth wear: systematic review. J Oral Rehabil. 2016;43(2):145-53. doi: 10.1111/joor.12360.

16. Demarco FF, Baldissera RA, Madruga FC, Simões RC, Lund RG, Correa MB et al. Anterior composite restorations in clinical practice: findings from a survey with general dental practitioners. J Appl Oral Sci. 2013; 21(6):497-504. doi: 10.1590/1679-775720130013.

17. Heintze SD, Rousson V, Hickel R. Clinical effectiveness of direct anterior restorations: a meta-analysis. Dent Mater. 2015;31(5):481-95. doi: 10.1016/j.dental.2015.01.015.

18. Rauber GB, Bernardon JK, Vieira LCC, Baratieri LN. Evaluation of a technique for color correction in restoring anterior teeth. J Esthet Restor Dent. 2017;29(5):309-16. doi: 10.1111/ jerd.12320.

19. Gouveia THN, Theobaldo JD, Vieira-Junior WF, Lima DANL, Aguiar FHB. Esthetic smile rehabilitation of anterior teeth by treatment with biomimetic restorative materials: a case report. Clin Cosmet Investig Dent. 2017;9:27-31. doi: 10.2147/ CCIDE.S130698.

20. Bazos P, Magne P. Bio-emulation: biomimetically emulating nature utilizing a histo-anatomic approach; structural analysis. Eur J Esthet Dent. 2011;6(1):8-19.

21. Haddad HJ, Salameh Z, Sadig W, Aboushelib M, \& Jakstat HA. Allocation of color space for different age groups using three-dimensional shade guide systems. Eur J Esthetic Dent. 2011;6(1):94-102.

22. Sproull RC. Color matching in dentistry. Part II. Practical applications of the organization of color. J Prosthet Dent. 2001;86(5):458-64. doi: 10.1067/mpr.2001.119829

23. Duarte S Jr, Perdigão J, Lopes M. Composite resin restorations: natural aesthetic and dynamics of light. Pract Proced Aesthet Dent. 2003;15(9):657-64.

24. Lee YK. Influence of scattering/absorption characteristics on the color of resin composites. Dent Mater. 2007;23(1):124-31. doi: 10.1016/j.dental.2006.01.007

25. Pecho OE, Ghinea R, do Amaral EA, Cardona JC, Della Bona A, Pérez MM. Relevant optical properties for direct restorative materials. Dent Mater. 2016:32(5):105-12. doi: 10.1016/j. dental.2016.02.008. 